\title{
Applications of Savitzky-Golay Filter for Seismic Random Noise Reduction
}

\author{
Yanping $\mathrm{LIU}^{1}$, Bo DANG ${ }^{1}$, Yue $\mathrm{LI}^{2}$, Hongbo $\mathrm{LIN}^{2}$, and Haitao MA ${ }^{2}$ \\ ${ }^{1}$ College of Electronic Engineering, Xi'an Shiyou University, Xi'an, China; \\ e-mail: liuyp09@gmail.com \\ ${ }^{2}$ College of Communication Engineering, Jilin University, Changchun, China
}

\begin{abstract}
This article utilizes Savitzky-Golay (SG) filter to eliminate seismic random noise. This is a novel method for seismic random noise reduction in which SG filter adopts piecewise weighted polynomial via leastsquares estimation. Therefore, effective smoothing is achieved in extracting the original signal from noise environment while retaining the shape of the signal as close as possible to the original one. Although there are lots of classical methods such as Wiener filtering and wavelet denoising applied to eliminate seismic random noise, the SG filter outperforms them in approximating the true signal. SG filter will obtain a good tradeoff in waveform smoothing and valid signal preservation under suitable conditions. These are the appropriate window size and the polynomial degree. Through examples from synthetic seismic signals and field seismic data, we demonstrate the good performance of SG filter by comparing it with the Wiener filtering and wavelet denoising methods.
\end{abstract}

Key words: SG filter, seismic random noise reduction, synthetic seismic signals, field seismic data.

\section{INTRODUCTION}

Estimating a signal of interest degraded by additive random noise is a classical problem in signal processing. In many applications, signal denoising is 
used to produce estimates of the original signal from noisy observations (Boudraa et al. 2004). Seismic data is always contaminated by various noise including coherent noise and random noise. The noise may distort and even cover the valid seismic signals. This is an obstacle for acquiring the useful information of seismic prospecting, which brings difficulties to geological interpretation. In this article, we mainly aim at seismic random noise reduction where we seek an effective method to extract seismic signals from noisy environments. Here, we introduce and test the capabilities of the so-called Savitzky-Golay (SG) filter.

SG filter was proposed by Savitzky and Golay in 1964 (Savitzky and Golay 1964). In the very beginning, this method was applied by the two authors in smoothing noisy data obtained from chemical spectrum analyzers, and it was demonstrated that least-squares smoothing reduces noise while maintaining the shape and height of waveform peaks (Schafer 2011). In recent years, some authors have adopted this method for reconstruction of Moderate-Resolution Imaging Spectrometer (MODIS) - (Enhanced Vegetation Index (EVI), Normalized Difference Vegetation Index (NDVI)) timeseries data (Chen et al. 2004, Huang et al. 2009, Bian et al. 2010, Li and Liu 2011), speech enhancement (Zehtabian and Hassanpour 2011), electrocardiogram (ECG) denoising (Hargittai 2005, Awal et al. 2011), pulse wave processing (Xu et al. 2011), remote sensing image merging (Chen and Shu 2011), among other applications, but it has not been applied to seismic random noise reduction yet.

SG filter which acts as a low-pass filter has a similar structure to a finite impulse response (FIR) filter. It is a method of data smoothing based on local least-squares polynomial approximation (Wayt and Khan 2007, Člupek et al. 2007). The smoothing can be controlled with two parameters, i.e., the window size and the polynomial degree. Small window sizes and high polynomial degrees may yield noisy signals ("flexible smoothing"), whereas large window sizes and low polynomial degrees may yield distorted signals ("rigid smoothing") (Vivó-Truyols and Schoenmakers 2006).

This method is simple and has a low computational cost. Additionally, some authors have proved that SG filter is superior in preserving valid signal to the moving average filter which belongs to the same kind of filters as SG in the same degree (Ruffin and King 1999, Vaiphasa 2006, Guiñón et al. 2007). Wiener filtering is a method based on the least-squares estimation too, but it requires the input process to be wide-sense stationary and its statistical characteristics should be known in advance ( $\mathrm{Li}$ and Bilgutay 1993, Nie 2012). However, these two conditions are difficult to be met in practical situations (such as seismic signal), which is why Wiener filtering does not always produces the best filtering result. Although the wavelet method is effective for seismic denoising and has been widely used in the geophysical 
field, SG filter is better than it in extracting the valid signal. That is because SG filter adopts local least-squares fitting of polynomials instead of global algorithm to approximate the true signal.

However, the restriction of SG filter mentioned by Vivó-Truyols and Schoenmakers (2006) reminds us that it could get excellent smoothing at the expense of losing small time-scale variations feature of signal by SG filter. Even though we adjust the window size and the polynomial degree to achieve an appropriate compromise between random noise reduction and valid signal preservation, the denoising ability of SG filter is still limited in a certain level in that it presents a difficult choice related to the trade-off between window size and degree of the polynomial. At this time, we could improve the filtering effect of SG filter by virtue of total variation (TV) regularization method (Chambolle 2004, Zhou and Li 2013).

In the following, we introduce SG filter and TV method in detail and demonstrate their capabilities through experiments on seismic signals.

\section{THE PRINCIPLE OF SG FILTERING}

\subsection{Savitzky-Golay (SG) filter}

SG filter is a simplified least-squares-fit convolution for smoothing and computing derivatives of a set of consecutive values. It can be understood as a weighted moving average filter with weight coefficients given as a polynomial of a certain degree (Chen et al. 2004). This method requires two key parameters: the window size and the polynomial degree. If the window length is too long, it will produce some loss of valid signals, whereas if the window length is too short, it cannot denoise well; if the polynomial degree is too high, it may yield redundant data and produce new noise, and if the polynomial degree is too low, it may yield over-smoothing and signal distortion. So, we should select the window length and the polynomial degree appropriately to achieve a good trade-off between random noise reduction and valid signal preservation. As a general rule, we select the polynomial degree ranging from two to six (we could select low degree under high SNR condition while select high degree under low SNR condition), and the window length could be adjusted by keeping the polynomial degree as a constant until obtaining an optimal result.

The mathematical description of the smoothing process implemented by SG filtering is shown by the formula:

$$
s_{j}^{*}=\frac{\sum_{i=-m}^{i=m} c_{i} s_{j+i}}{N},
$$


where $s$ is the original signal, $s^{*}$ is the smoothed signal, $c_{i}$ is the coefficient for the $i$-th smoothing, $N$ is the number of data points in the smoothing window and is equal to $2 m+1$, where $m$ is the half-width of the smoothing window. The index $j$ represents the running index of the ordinate data in the original data table (Chen et al. 2004, Bian et al. 2010, Li and Liu 2011). The essence of SG filtering is adopting a polynomial in a sliding window to fit the original signal piece-by-piece depending on the least-squares estimation algorithm. The polynomial can be modeled as:

$$
f_{k}(i)=b_{0}+b_{1} i+b_{2} i^{2}+\cdots+b_{k} i^{k}=\sum_{n=0}^{k} b_{n} i^{n}, \quad i \leq 2 m .
$$

Here, $b_{n}$ are the coefficients of this polynomial, and $k$ denotes the polynomial degree. A major characteristic of SG filter is that it combines differentiation and smoothing into one algorithm, which is sensible because smoothing is always required with differentiation (O'Haver 2012, 2013). So, SG filter can implement smoothing by high order differentiation but the differentiation of each order plays a different role. However, we usually adopt a zero order differentiation smoothing to remove random noise from the original noisy signal.

In SG algorithm, the least-squares criterion expressed by Eq. 3 is applied to obtain the unknown filtering coefficients $b_{n}$.

$$
\frac{\partial}{\partial b_{n}}\left[\sum_{i=-m}^{m}\left(f_{k}(i)-s_{i}\right)^{2}\right]=0 .
$$

This leads to $k+1$ simultaneous equations for computing the unknown coefficients $b_{n}$. We evaluate Eq. 2 at $i=0$ and can only obtain an expression for $b_{0}$. Then, we compute the $n$-th differential of Eq. 2 at $i=0$ to get $b_{n}$. Here, the obtained coefficients $b_{0}, b_{1}, \ldots, b_{k}$ are equivalent to the desired coefficients $c_{i}, i \in[-m, m]$. So, we deduce an expression from Eq. 2 as:

$$
f_{k}^{n}(0)=\sum_{i=-m}^{m} c_{i}^{n} s_{i},
$$

where $n$ denotes the derivative order, $c_{i}^{n}$ and $s_{i}$ are the convolution weight and the value of $i$-th point, respectively.

As the contents we mentioned previously, SG filter can obtain a good trade-off between valid signal preservation and random noise reduction. However, there are still some problems that need to be addressed. The unsatisfactory phenomenon caused by SG filter is different from other algorithms consisting in the details mainly about the peaks and spikes produced somewhere on the filtered waveform in a relatively heavy noise environment. 
Thus, there may be a big temptation of applying iterative filtering to SG filter like some multiple filtering algorithms. To be specific, if the result of the first time filtering is unsatisfactory, it seems that we could do several times filtering to get smoother waveform, but it must cause serious losses for the valid signal. Although we could select shorter window length for the following times filtering, there is still a loss for the valid signal which could not be ignored. So, we do not intent to achieve smoothing through iterative filtering by SG filter.

\subsection{SG filtering based on TV regularization criterion}

The total variation (TV) method has been introduced in computer vision first by Rudin et al. (1992). It is as a regularizing criterion based on a gradient energy function for solving inverse problems (Chambolle 2004). It can measure how much the signal changes between signal values. Normally, the $\mathrm{TV}$ of a noisy signal is obviously large, so we can obtain the denoised signal through minimizing the TV of the noisy signal.

A noisy signal can be modeled as:

$$
s_{\text {noisy }}(t)=x_{\text {desired }}(t)+\operatorname{noise}(t) .
$$

The minimization criterion represented by $J$ of TV denoising method is expressed as follows:

$$
J(s)=\min \left\{\|D s\|_{1}+(\lambda / 2)\|s-x\|_{2}^{2}\right\},
$$

where $s$ denotes the noisy signal, $x$ denotes the desired signal, and $D$ represents the first order differential form. $\lambda$ is a regularization factor which is any real number greater than zero. The first item which is also the total variation of $s$ is the 1-norm item. The second item is the 2-norm item (or the fidelity item) which adopts the least-squares estimation criterion too.

So far, the TV method has been often used to eliminate noise, especially for image processing (Vogel and Oman 1996, Chambolle and Lions 1997, Chan et al. 2006, Chambolle et al. 2010, Bonettini and Ruggiero 2011, Chen and Cheng 2012, Zhou and Li 2013). Some people also apply this method to seismic random noise reduction (Qu et al. 2011, Gong 2012). The TV-based denoising techniques adopt a variational method to deduce a group of partial differential equations (PDE) which possess initial conditions and boundary conditions. Those are then solved to obtain the denoised signal using numerical computation methods.

Although the TV method can be used to denoise, there is a drawback quite necessary to overcome, that is, the phenomenon of "staircase effect" (Dong and Liu 2009, Selesnick and Bayram 2010). The "staircase effect" re- 
fers to the waveform looked like staircase, which is caused by the TV method when it processes the continuous and smooth signals. In other words, the TV method allows for the uncontinuity of signals. The severity degree of this phenomenon is attributed to a parameter of the TV method which is the regularization factor $\lambda$. The selection of its value depends on the noise level. The larger the noise, the larger the $\lambda$. In fact, the TV method shows good denoising ability in processing the signals similar to the blocks signal of presenting piecewise constant (Vogel and Oman 1996). Nevertheless, most practical signals are nonlinear, non-stationary, and some are continuous. So, the larger value of $\lambda$ could lead to effective noise removal when we apply the $\mathrm{TV}$ denoising method to process these signals, but the detailed information of the valid signals will be lost a lot due to the "staircase effect". Therefore, if we want this method to work better in a wide range of applications, we should bring some measures which are conducive in preserving the details of valid signals into the whole filtering procedure. Here, the SG filtering principle is brought in.

Since the seismic signal is nonlinear and non-stationary, there are some difficulties to track its variation trend generally. For example, the Wiener filtering belongs to linear methods which are not so effective when signals contain sharp edges and pulses of short duration. A main drawback of the wavelet approach is that the basis functions are fixed, and do not necessarily match the varying nature of signals (Boudraa et al. 2004). In contrast, the SG filter is a commendable method for processing non-stationary signals. That is because it possesses corresponding characteristics to track the variation trend of seismic signals and could extract the valid seismic signals effectively.

The SG filter applies a local weighted polynomial fitting method via a least-squares criterion to achieve smoothing. So, it could remove the random noise while retaining the small scale features for the original signal. That is to say, it could retain the shape of the original signal as much as possible while preserving its fidelity.

However, the capability of SG filter is limited by the window size and the polynomial degree which we have analyzed previously. It will obtain comparatively good results only in the case of achieving a compromise between the two aspects just mentioned.

With respect of this restriction, the denoising ability of SG filter is limited in a certain level. Taking the synthetic seismic signals which are our experimental objects shown in the following figures for example, there are non-zero and non-linear sections combined with zero sections in the original noise-free signals. SG filter could extract the non-zero and non-linear sections of the original signals very well from the noisy environments, while it could not perform so well for the zero sections. When the noise is quite heavy, it produces some peaks and spikes on the filtered waveform, especial- 
ly at places corresponding to the zero value sections of the original signal. This occurs even under the appropriate condition of a good trade-off between the window size and the polynomial degree of SG filter. So, we try to enhance the denoising ability of SG filter by virtue of TV regularization criterion, particularly for the peaks and spikes produced in the heavy noise environment. Thus, we could utilize the respective advantages of SG and TV to eliminate the random noise better as well as preserve the original valid signal.

Our method is designed to bring the principle of SG filtering into TV regularization criterion. It can be expressed by the following formula:

$$
J\left(s_{j}^{*}\right)=\min \left\{\left\|D s_{j}^{*}\right\|_{1}+(\lambda / 2)\left\|s_{j}^{*}-x\right\|_{2}^{2}\right\} .
$$

In fact, the whole filtering process still applies the least-squares estimation. We only combine the two factors including the local polynomial fitting provided by SG filter and the gradient energy function provided by TV method to this process. Hence, the filtering capability of SG filter will be enhanced. For example, a synthetic seismic signal simulated by Ricker wavelet is piecewise non-linear, non-stationary, and piecewise linear (or constant), which is shown in the following text. For giving extended applications, we can adopt this improved method to process the signals like the synthetic seismic signal to obtain better filtering results than only adopt SG filter. But for field seismic signals, there are some differences with the synthetic seismic signal experimented here in that there are almost non-zero sections in the waveform. The signal presents strong non-linearity and non-stationarity, and the field seismic random noise is not evenly distributed. Considering the above, we experiment on both synthetic seismic models and field seismic data to test each method.

\section{SEISMIC SIGNAL PROCESSING}

We do experiments on both synthetic seismic models and field seismic data by SG filter. We then compare the results with Wiener filtering and wavelet denoising methods. The noisy seismic signal also can be modeled as formula 5 in which $s_{\text {noisy }}(t)$ denotes the noisy seismic signal, $x_{\text {desired }}(t)$ denotes the valid component of the seismic signal, and noise $(t)$ denotes an additive random noise (additive white Gaussian noise (AWGN) is the simplest situation). First, we take a noisy Ricker wavelet with the signal to noise ratio (SNR) of about $10 \mathrm{~dB}$ to test the Wiener filtering, wavelet denoising and SG filtering, respectively. Referring to some references listed at the end of this article, combined with numerous experiments, we set the order of Wiener filter to be 12, the degree and the window length of SG filter to be 2 and 37, 


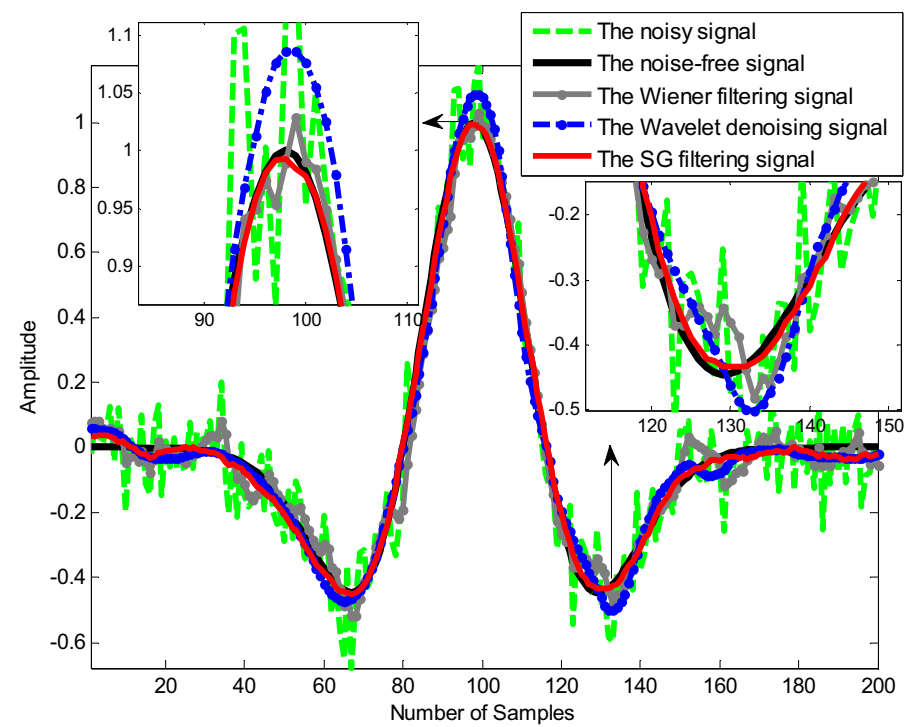

(a)

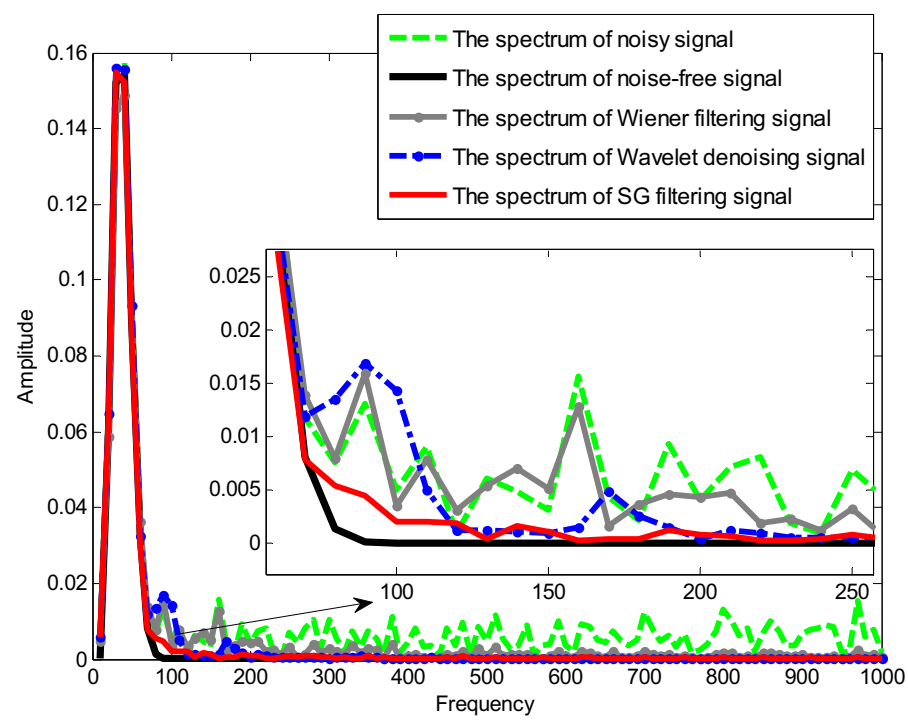

(b)

Fig. 1. Comparisons of the waveforms and spectra for an experiment performed on a noisy Ricker wavelet with a SNR of about $10 \mathrm{~dB}$. Panel (a) shows that the waveform of the Wiener filtering is the most unsmoothed among the three filtered signals. In particular, the waveform of the wavelet denoising signal is smoother than the one from the Wiener filtering, but it has larger deviations compared to the noise-free signal. The SG filtering signal is the best approximate to the noise-free signal among the three filtering signals. Panel (b): in the Fourier spectra of the five signals shown in (a), the SG filtering signal is more flat than the spectrum of the Wiener and wavelet filtering signals. 
respectively, and the type of wavelet to be $5 \mathrm{~dB}$ with soft threshold for this experiment.

The resulting comparisons of waveforms and spectra of the noisy signal, the noise-free signal, the Wiener filtering signal, the wavelet denoising signal, and the SG filtering signal are shown in Fig. 1.

It can be found that the waveform filtered by SG filter is more similar to the ideal signal than the waveforms obtained by Wiener filtering and wavelet denoising methods, especially in the wave peak and troughs. Moreover, the wavelet denoising result is better than the Wiener filtering result. The spectrum of SG filtering result is closer to the spectrum of the ideal signal than both the spectra of Wiener filtering and wavelet denoising signals. These observations are highlighted by the enlarged fragments in (a) and (b) of Fig. 1. This experiment demonstrates the good performance of SG filter in random noise reduction and valid signal preservation under a comparatively high SNR circumstance. In addition to the qualitative analysis, we also give quantitative analysis through computing the SNR and mean square error (MSE) of the four signals shown in Table 1. The computation methods of SNR and MSE refer to Ferahtia et al. (2010), which are represented as follows:

$$
\begin{gathered}
\mathrm{SNR}=10 \log _{10} \frac{\sum_{i=1}^{N} \sum_{j=1}^{M} x(i, j)^{2}}{\sum_{i=1}^{N} \sum_{j=1}^{M}\left(s^{*}(i, j)-x(i, j)\right)^{2}}, \\
\mathrm{MSE}=\frac{1}{N M} \sum_{i=1}^{N} \sum_{j=1}^{M}\left(s^{*}(i, j)-x(i, j)\right)^{2},
\end{gathered}
$$

where $s^{*}$ denotes the filtered data, $x$ denotes the noise-free data, $N$ denotes the number of samples, and $M$ denotes the number of traces.

Table 1

\begin{tabular}{|c|c|c|c|c|}
\hline Signals & Noisy signal & $\begin{array}{c}\text { Wiener filtering } \\
\text { signal }\end{array}$ & $\begin{array}{l}\text { Wavelet denoising } \\
\text { signal }\end{array}$ & $\begin{array}{c}\text { SG filtering } \\
\text { signal }\end{array}$ \\
\hline SNR & & & & \\
\hline MSI & 0.0092 & 0.0028 & 0.0017 & 0.00036 \\
\hline
\end{tabular}

The SNR and MSE of the filtered signals

We just have illustrated the characteristic of SG filter that it shows good smoothing and preserving abilities under a relatively high SNR condition. We have also proposed the limitation of SG filter that its smoothing ability will be restricted in a certain level under a relatively low SNR condition. 
That is to say, when the background noise is heavier, SG filter could not denoise very well and some peaks and spikes will appear on certain areas of the filtered waveform. However, its preserving ability for valid signal is still good in this situation. At this time, we want to enhance the denoising ability of SG filter by virtue of some measures which are good at smoothing the peaks and spikes.

We attempt to utilize TV method to assist SG filter in better eliminating the random noise. The idea of SG filtering based on TV criterion just combines the respective advantages of SG filter and TV method. Hence, it cannot only preserve the valid signal but also can better denoise, especially in heavy noise environments.

Next, we experiment on a synthetic seismic model which is composed of two reflection events. The dominant frequencies of the events are 30 and $25 \mathrm{~Hz}$, respectively. The velocities are 1900 and $2200 \mathrm{~m} / \mathrm{s}$, respectively. This model has $1 \mathrm{~ms}$ sampling with 100 traces of 1500 points each. We add AWGN to it to make the SNR to be about $5 \mathrm{~dB}$. We apply Wiener filtering, wavelet denoising, SG filtering, and SG-TV filtering methods to denoise this record. We set the order of Wiener filter to be 12, and select $5 \mathrm{~dB}$ wavelet with soft threshold. Since the SNR is lower than the SNR of the noisy signal shown in Fig. 1, we need to set a higher order polynomial for SG filter to fit the ideal signal. So, we set the degree and the window length of SG filter to be 6 and 37, respectively, in processing this noisy record. The filtering results are shown in Figs. 2 to 6.

From visual inspection of Fig. 3 it can be seen that the denoising effect is almost the same between the SG filtering record and the wavelet denoising record while the Wiener filtering record is the worst.

From Fig. 4 it can be found that there are almost no reflection events in the difference between the noisy record and the filtered record by SG filter. In contrast, the residual reflection events are obviously present in the difference between the noisy record and the Wiener filtering record. Although there may be almost no reflection events in the difference record between the noisy record and the wavelet denoising record, the difference record presents larger deviation compared to the original noise record. The obvious parts of each difference record are boxed in white rectangles.

In other words, since the difference between the noisy record and the SG filtering record is most similar to the original noise record, it demonstrates that SG filter provides the highest fidelity for the original valid signals among these three methods.

In fact, even if the noise is stronger than in the first experiment, SG filter still presents good extraction for the non-zero value parts of the original signals. Just a slightly unsmooth phenomenon appears on the waveform, especially in the zero value parts of the original signals. This point will be shown 


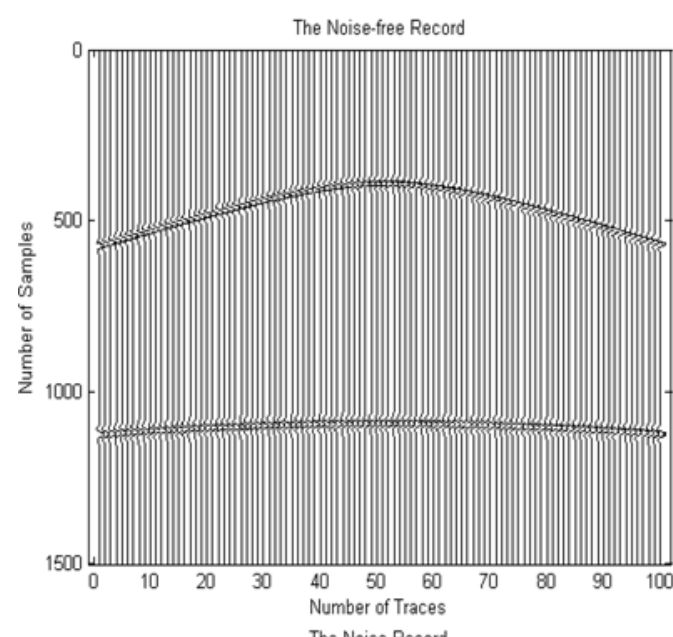

(a)

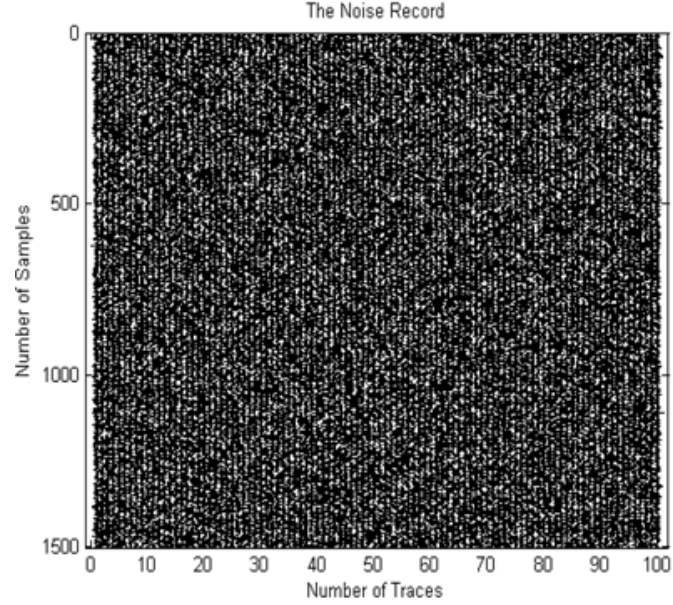

(b)

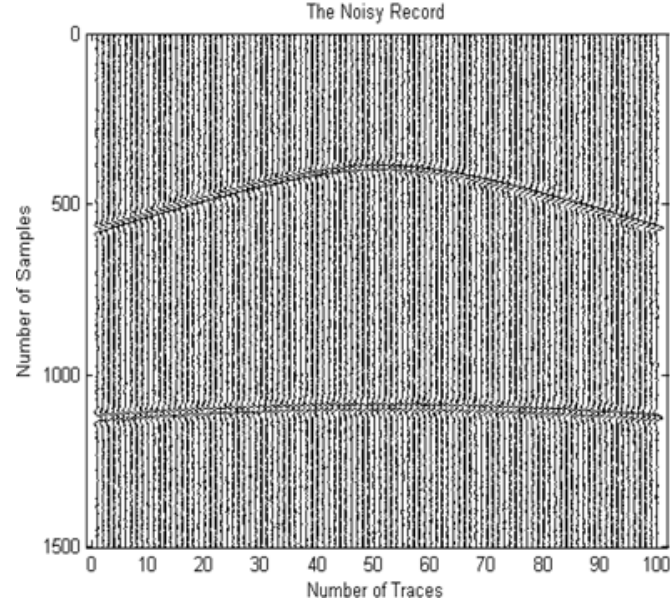

(c)

Fig. 2. In our synthetic example, we consider a seismic record containing two reflection events (a) to which we then add noise (b) in order to obtain a noisy data set (c). 


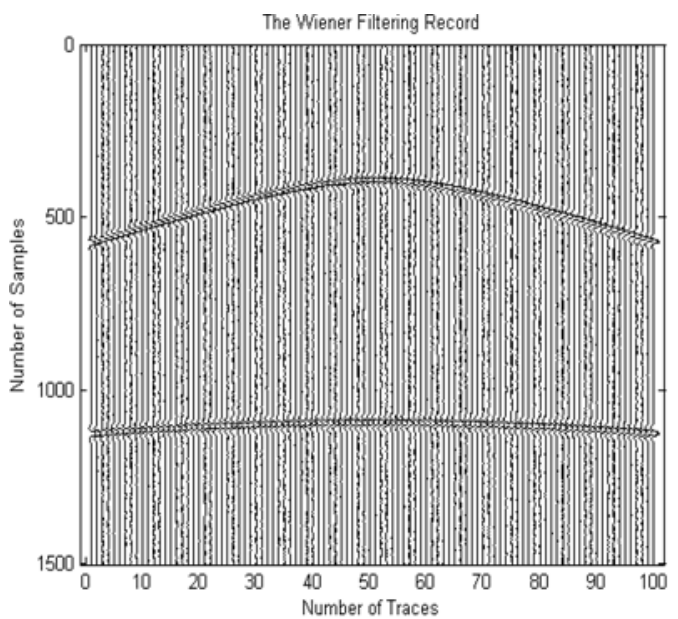

(a)

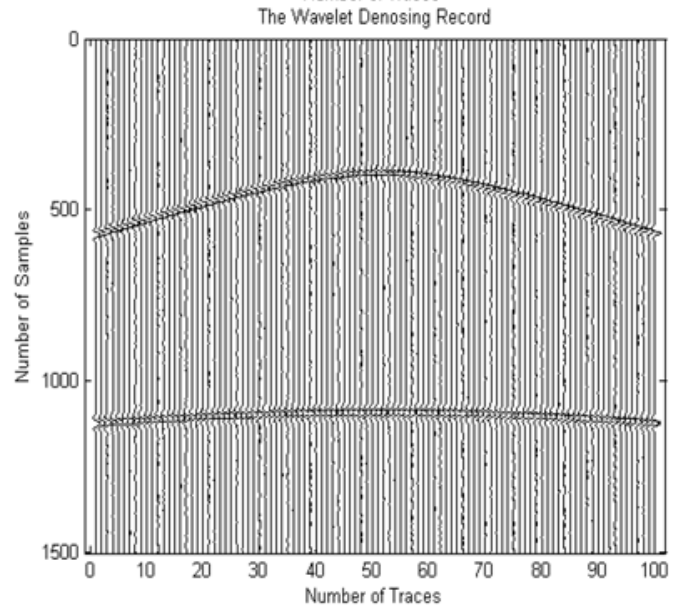

(b)

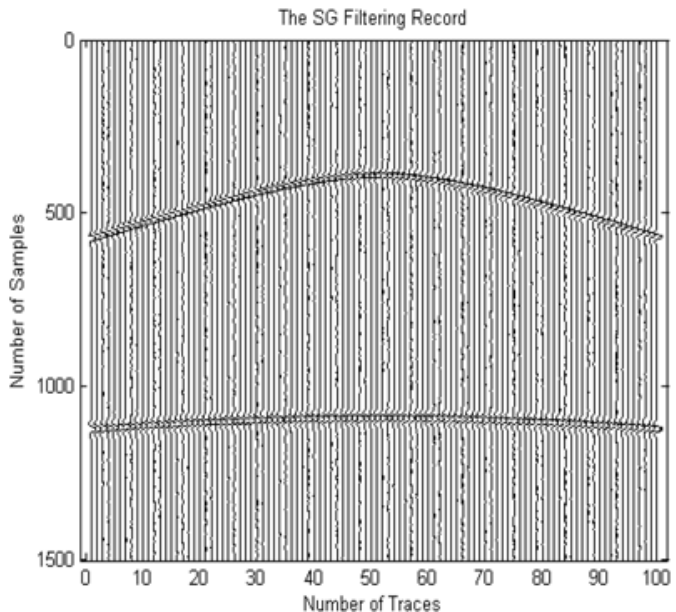

Fig. 3. Comparison of different filters applied to the noisy synthetic seismic record shown in Fig. 2c. In the Wiener filtering record, there is still much noise, while in the wavelet denoising record, the residual noise is less than in the Wiener filtering record. In contrast, for the SG filtering record, the residual amount of background noise is almost the same as in the wavelet denoising record. 


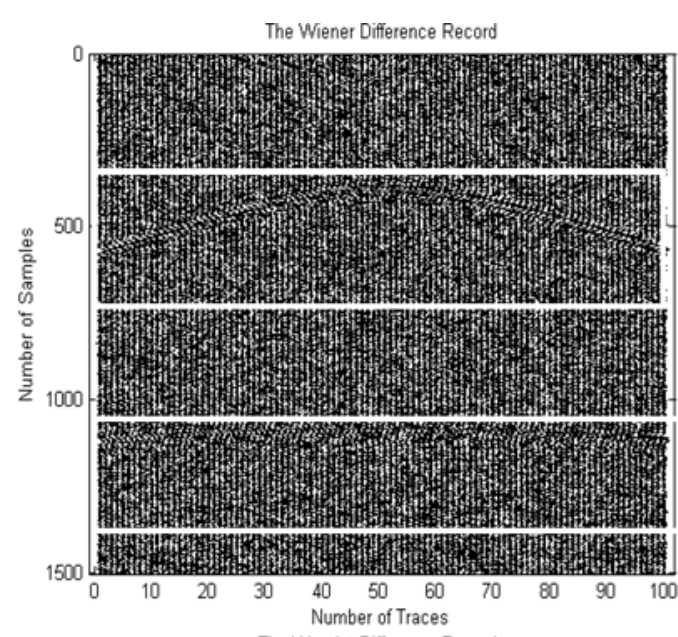

(a)

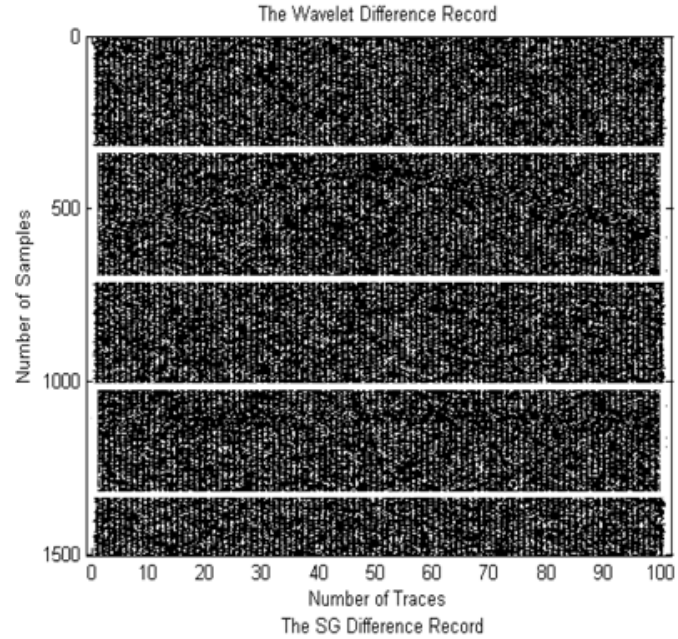

(b)

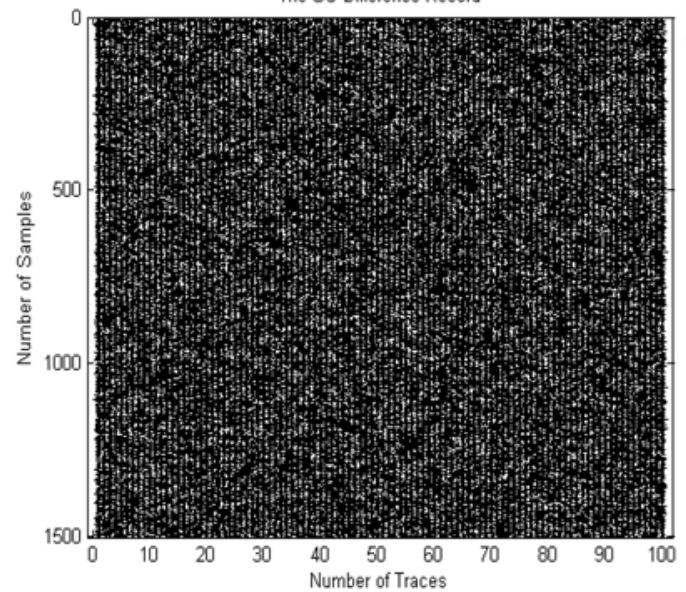

Fig. 4. Comparison of difference records between the noisy record and the filtered records. In the Wiener difference record, parts of valid events are lost in this record, while the loss of valid events (c) in the wavelet difference record was lower than in the Wiener difference record. However, the bias of wavelet difference record is so large compared with the original noise record that it could not be neglected. In contrast, the least valid events residue in the SG difference record and this record is the best approximate to the original noise record among the three difference records. 

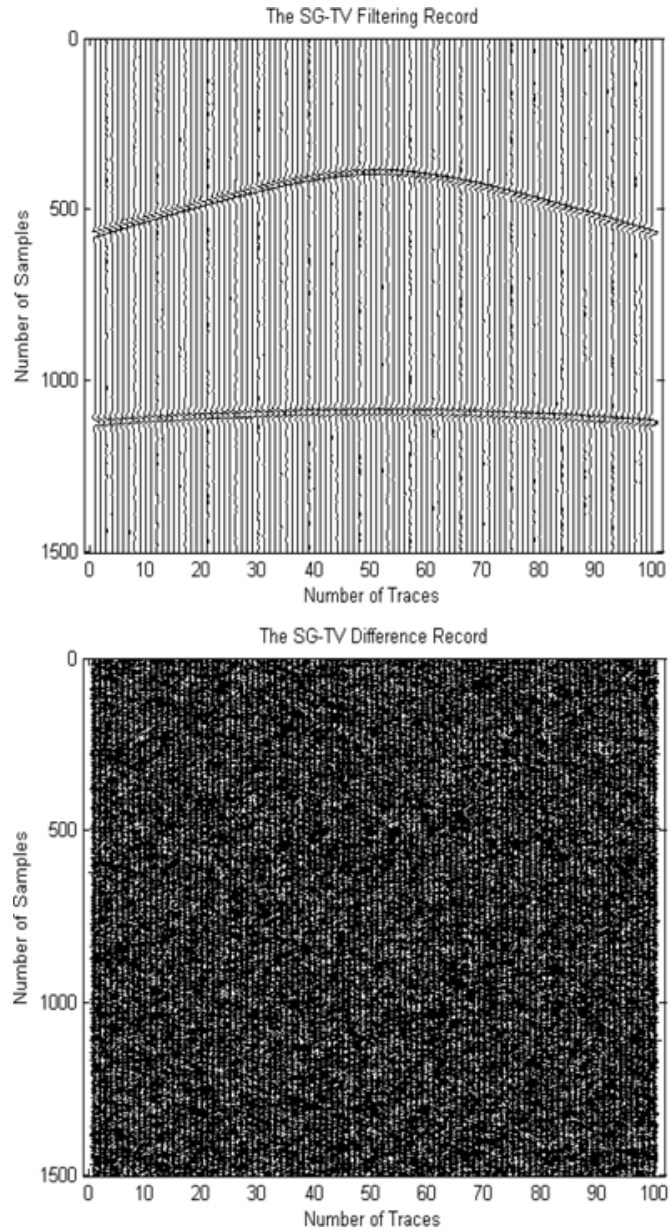

(a)

(b)

Fig. 5. SG-TV method is applied to the noisy synthetic seismic record shown in Fig. 2c. Panel (a) shows the SG-TV filtering record. We subtract (a) from the noisy record to obtain the difference record (b). To compare this difference record with the noise record shown in Fig. 2b, we can see that these two records are almost the same.

in Fig. 6. If the valid signal is seriously contaminated by random noise, the unsmooth phenomenon will be obvious and we need to improve this problem. So, we experiment on the same noisy record shown in Fig. 2c by SGTV filtering method and the experimental results are shown in Fig. 5.

It can be found that the background noise has been removed more, and there is almost no reflection events left in the difference record too. This point demonstrates that the TV method almost attenuates the not valid signal in the whole smoothing process. For this, we have to select a suitable value for the regularization parameter $\lambda$.

We also compare the waveforms derived from a certain channel of the noisy record, the noise-free record, the Wiener filtering record, the wavelet denoising record, the SG filtering record, and the SG-TV filtering record, respectively. In detail, we also show the waveform comparisons corresponding 
to a section of the original signal with zero values for shallow and deep layer signals, respectively. Figure 6 shows the single channel waveform comparisons.

Similar to the results from Fig. 1, we find that SG-based methods are the closest to the original valid signal, especially in the wave peaks and troughs. In this experiment, the TV method performs more obvious smoothing for the

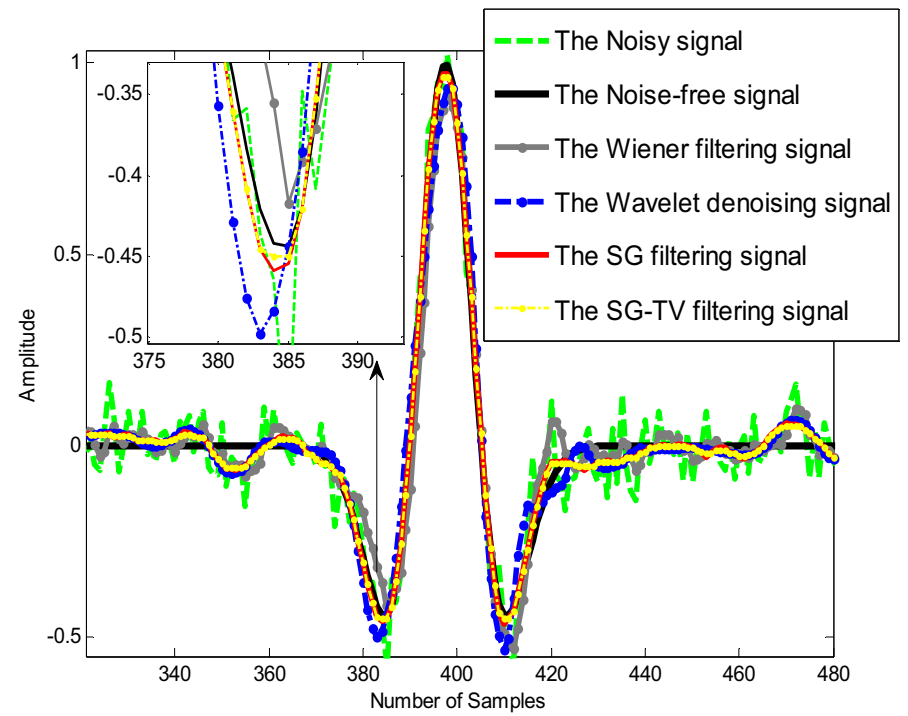

(a)

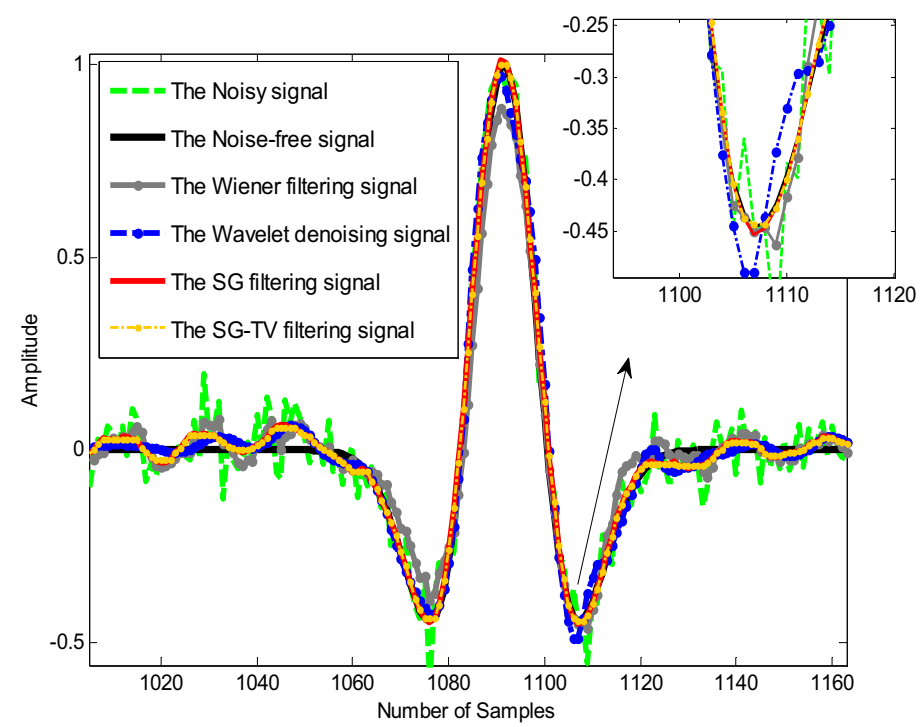

(b)

Fig. 6. Continued on next page. 


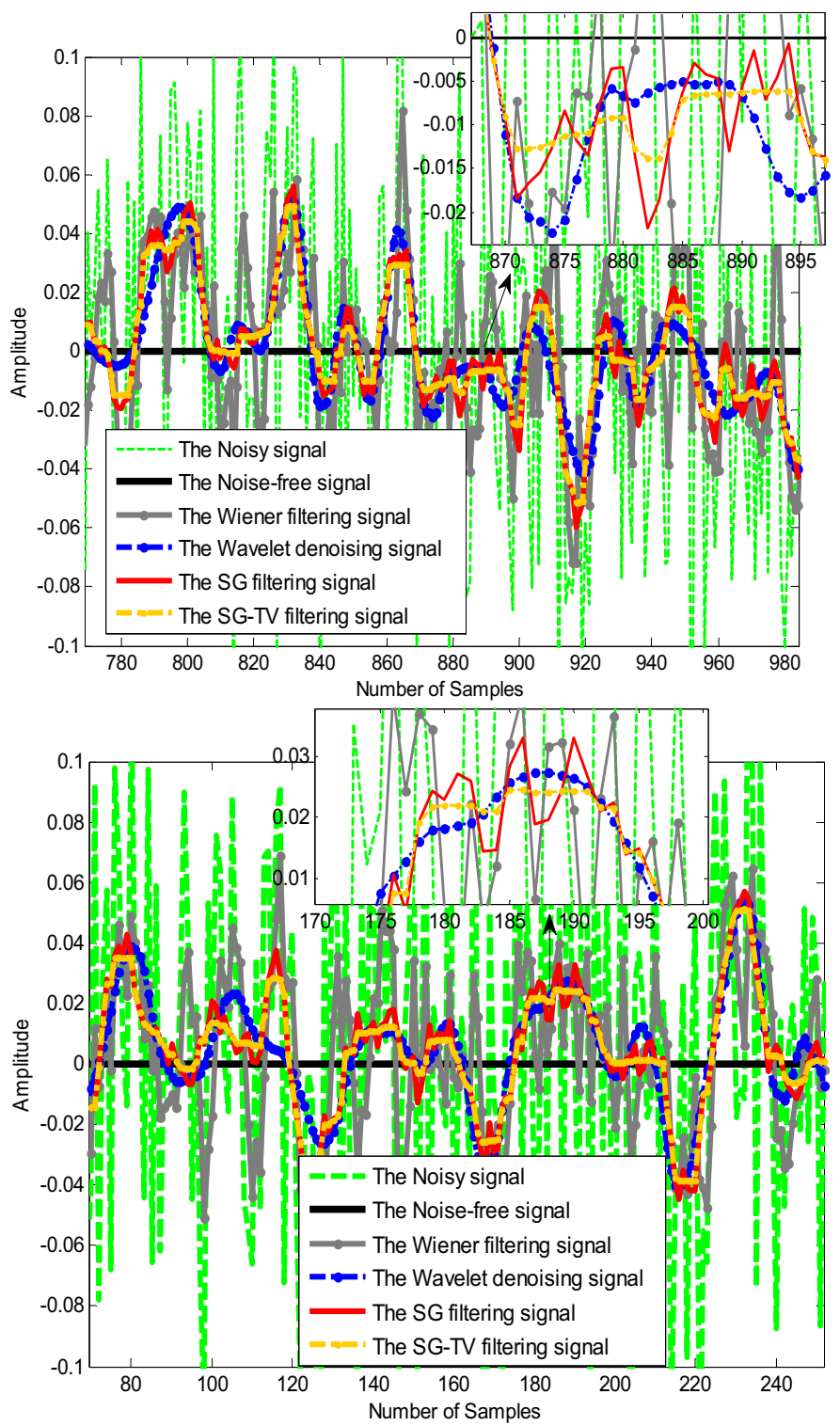

(c)

Fig. 6. The waveform comparisons and enlarged figures. Panel (a) shows the waveform comparison of the shallow layer Ricker wavelet. We enlarge parts of the waveforms in (c). Panel (b) shows the waveform comparison of the deep layer Ricker wavelet. We enlarge parts of the waveforms in (d). The Wiener filtering signal presents a seriously unsmooth signal, while the wavelet denoising signal is smoother than the Wiener filtering signal. The waveforms of SG and SG-TV filtering methods are the best approximates to the noise-free signal among these four filtering signals, while the SG-TV filtering signal is smoother than the SG filtering signal. 
zero value parts than non-zero value parts of the original valid signals with stronger noise. If the noise is much stronger, the smoothing effect of TV method will be obvious for the whole signal.

We also compute the SNR and MSE of the noisy record, the Wiener filtering record, the wavelet denoising record, the SG filtering record, and the SG-TV filtering record, respectively (Table 2).

Table 2

The SNR and MSE of the filtered records

\begin{tabular}{|c|c|c|c|c|c|}
\hline Records & Noisy record & $\begin{array}{c}\text { Wiener } \\
\text { filtering } \\
\text { record }\end{array}$ & $\begin{array}{c}\text { Wavelet } \\
\text { denoising } \\
\text { record }\end{array}$ & $\begin{array}{c}\text { SG } \\
\text { filtering } \\
\text { record }\end{array}$ & $\begin{array}{c}\text { SG-TV } \\
\text { filtering } \\
\text { record }\end{array}$ \\
\hline SNR [dB] & $4.9948 \mathrm{e}+00$ & $1.0164 \mathrm{e}+01$ & $1.2643 \mathrm{e}+01$ & $1.3856 \mathrm{e}+01$ & $1.4274 \mathrm{e}+01$ \\
MSE & $4.3000 \mathrm{e}-03$ & $1.3000 \mathrm{e}-03$ & $7.4060 \mathrm{e}-04$ & $5.6014 \mathrm{e}-04$ & $5.0873 \mathrm{e}-04$ \\
\hline
\end{tabular}

Third, we apply both the wavelet denoising and the SG filtering methods to process a field seismic data which has been acquired from an oilfield in China. This is a common shot-point (CSP) record of $4 \mathrm{~ms}$ sampling with 5480 samples of each channel. We will not consider the Wiener filtering method since it is more applicable to stationary signals. We have tested on synthetic seismic signals that its denoising ability is inferior to the abilities of wavelet denoising and SG filtering methods for non-stationary signals. Moreover, in practical applications, the field seismic signal has almost no zero value parts, and the random noise is not very strong in the whole record but in local areas. So, the SG filter can play the best role in keeping the fidelity of the reflection events while eliminating random noise. We set the corresponding parameters for each method according to the experiments on synthetic seismic records to process the field record.

The experimental results are shown in Figs. 7 to 10. In Fig. 7, we can find that the SG filtering method is superior to the wavelet denoising method in seismic signal processing. This mainly manifests in the aspects that the background noise is removed more and the valid reflection events are clearer and more continuous. The obvious areas are marked by white ovals and rectangle boxes in these three records.

In order to see clearly, we zoomed in the right part marked by white rectangle box of each record and show the enlarged drawing in Fig. 8.

To analyze the results in more detail, we make waveform comparisons of certain channel signals derived from the original CSP record, the wavelet denoising record and the SG filtering record, respectively. Figure 9a shows a waveform comparison of a certain channel in which the seismic signal is contaminated by a large amplitude noise. Figure $9 \mathrm{~b}$ shows a waveform com- 


\section{Original record}

Wavelet denoising record

SG filtering record

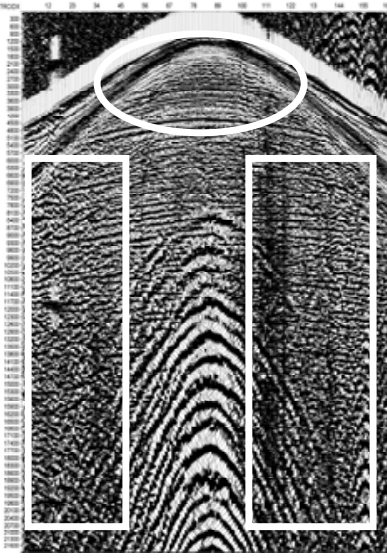

(a)

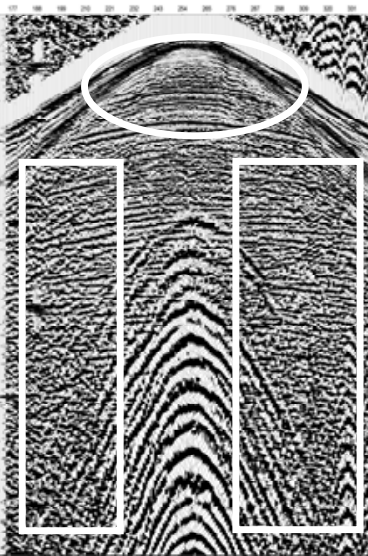

(b)

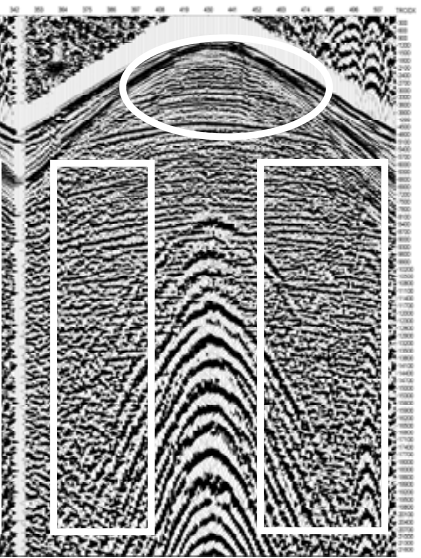

(c)

Fig. 7. Comparison of different filters applied to a field seismic record. In the wavelet denoising record, the residual noise is stronger than in the SG filtering record. The comparison of filtering effect is obvious in the top part marked by white oval and the left part marked by white rectangle box of each record. In the oval of panel (c), the reflection events are clearer than in (b), and in the left rectangle box of (c), the block noise is attenuated much more than in (b).

Original record

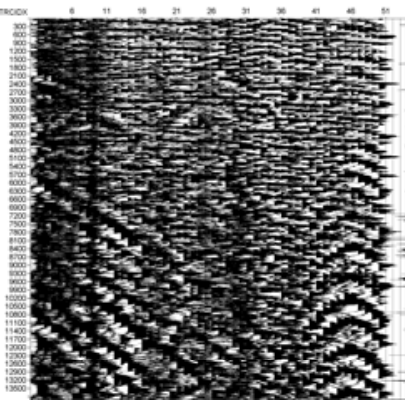

(a)

Wavelet denoising record

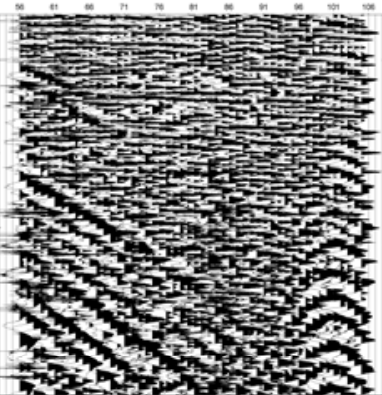

(b)
SG filtering record

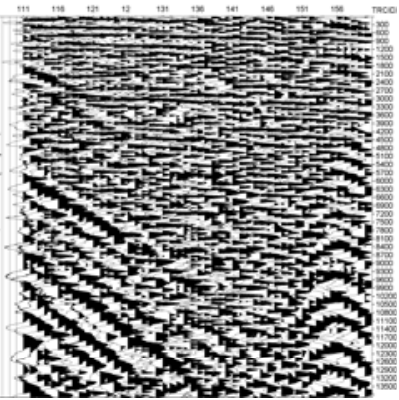

(c)

Fig. 8. The enlarged fragment of the right part of each record marked by white rectangle box is shown in Fig. 7. We can find that although both wavelet filter and SG filter can attenuate the random noise effectively, there is more residual noise in the wavelet denoising record while the reflection events are clearer in the SG filtering record.

parison of a certain channel in which the seismic signal is contaminated by a relatively homogeneous noise. We can see that the SG filtering signals are smoother than the wavelet denoising signals. This proves again that SG filter 


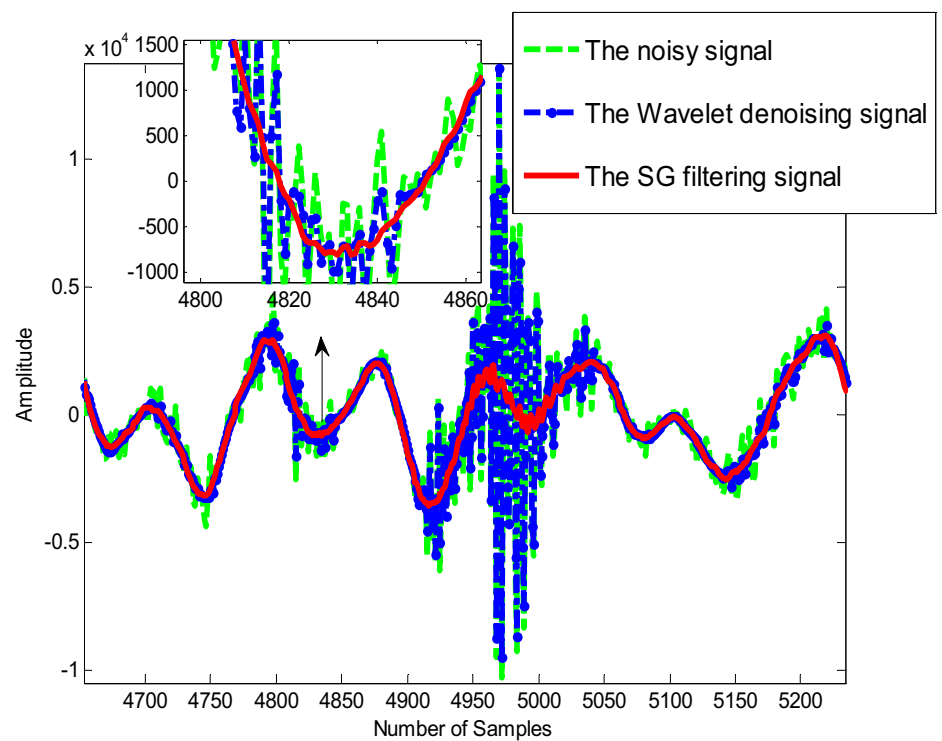

(a)

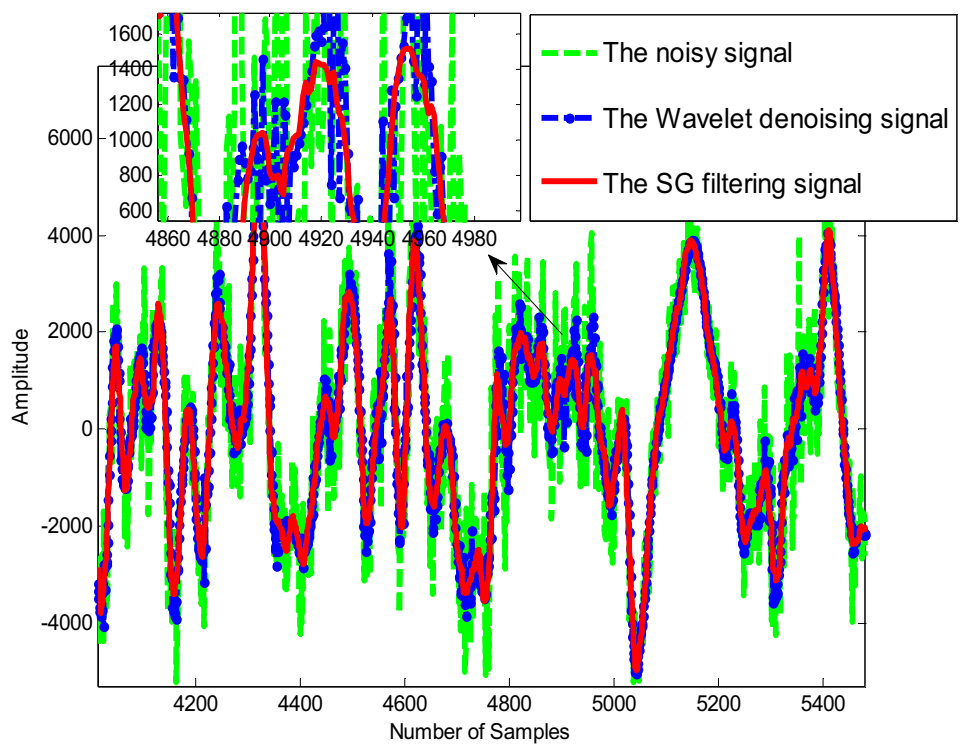

(b)

Fig. 9. The waveform comparisons and parts of enlarged figures for single channel signals derived from the three records. Panel (a) shows the waveforms of channel 16 in which the seismic signal is contaminated by large amplitude noise somewhere. In this situation, the wavelet denoising signal is very unsmooth in the heavy noise part while the SG filtering signal is quite smooth. Panel (b) shows the waveforms of channel 131 in which the seismic signal is contaminated by the noise with relatively homogeneous distribution. The signal of the SG filtering method is also smoother than the signal of the wavelet denoising method. 


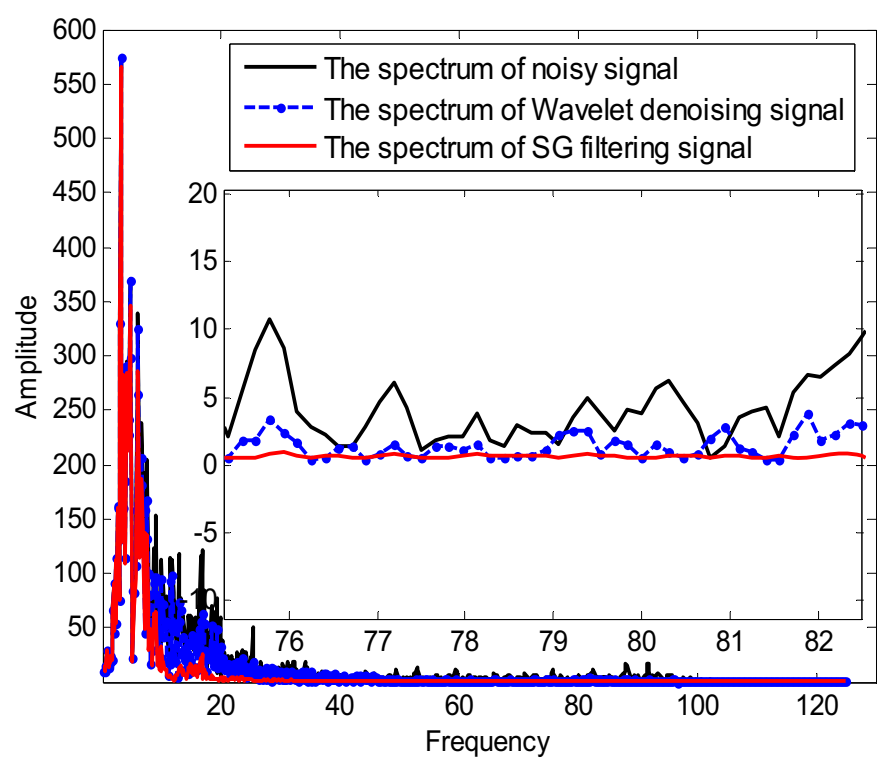

(a)

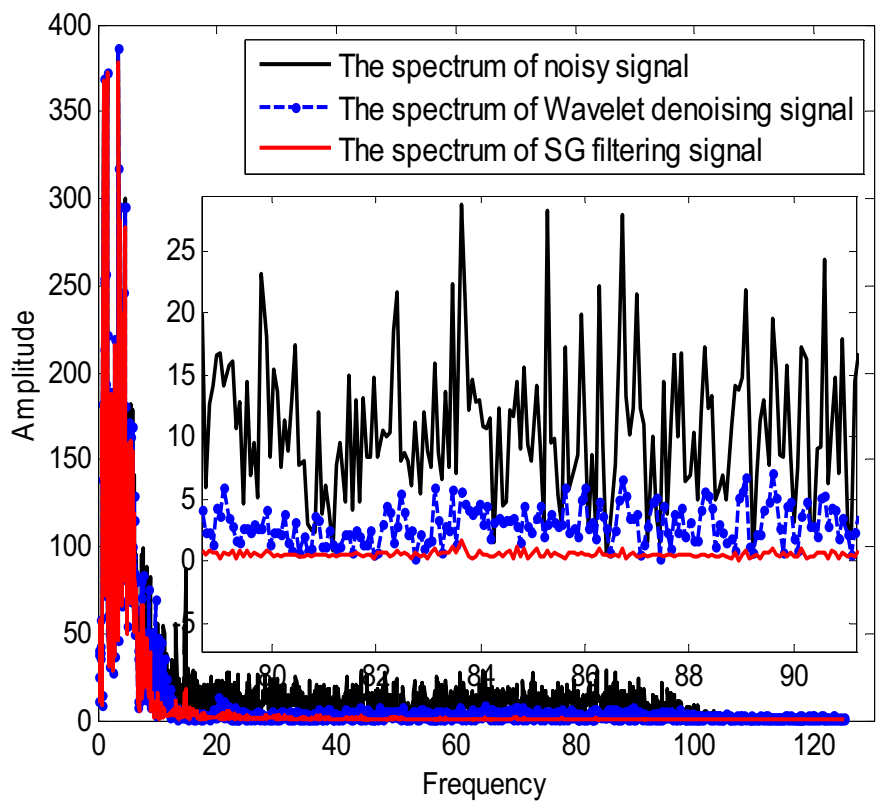

(b)

Fig. 10. The spectra comparisons and enlarged figures for single channel signals derived from the three records. Panel (a) shows the spectra of the upper part of channel 85 , and (b) shows the spectra of the lower part of channel 131. In both figures, the spectrum of the SG filtering signal is more flat than the spectrum of the wavelet denoising signal. 
is more suitable for processing non-stationary signals than wavelet denoising method.

Finally, the spectrum analysis is carried out by computing the Fourier spectrum of the signals derived from certain channels of the original record, the wavelet denoising record and the SG filtering record, respectively. In order to verify the denoising effect of wavelet filter and SG filter in the upper part of each record, we select channel 85 to do the spectra comparison for the corresponding part. Similarly, we select channel 131 to do the corresponding spectra comparison to verify the denoising effect of the two methods in the lower part of each record. From the following two figures, it can be seen that the spectrum of the SG filtering signal is more flat than the spectrum of the wavelet denoising signal, especially in the high frequency part. So, we can say that the SG filter is more effective in random noise reduction than the wavelet filter.

\section{DISCUSSION AND CONCLUSION}

SG filter is a novel tool for seismic processing and we have applied this method to eliminate seismic random noise and recover the valid seismic signals. It shows superior capability in extracting valid signals from noisy environments, especially in retaining the shape of the original signals. This method has broader applicability, and is particularly applicable for processing non-linear and non-stationary signals such as seismic signals.

However, SG filtering may produce unsmooth phenomena in very strong noise environments. According to this situation, we desire to enhance the denoising ability of SG filter with the aid of TV regularization criterion. Thus, it can make full use of their respective advantages and will be applicable for the signals presenting piecewise non-linearity and non-stationarity along with piecewise linearity. We have tested this measure on synthetic seismic signals simulated by Ricker wavelet and have obtained encouraging results. We intend to develop the universal applicability of this method in future studies.

In conclusion, through experiments on synthetic seismic signals and field seismic data, we demonstrated that SG filter possesses commendable performance in keeping the fidelity of valid seismic signals as well as eliminating seismic random noise compared with Wiener filtering and wavelet denoising methods. However, there is still a lot of room for the improvement of limitations in SG filter and SG-TV filter, and we need to try the best effort to do related works in the future.

Acknowledgements. We would like to thank the members of modern signal processing laboratory of Jilin University in China. This work is 
supported by National Natural Science Foundation of China with the grant no. 41174160, 41130421 and 41274118, respectively.

\section{References}

Awal, Md.A., S.S. Mostafa, and M. Ahmad (2011), Performance analysis of Savitzky-Golay smoothing filter using ECG signal, Int. J. Comput. Inf. Technol. 1, 2, 24-29.

Bian, J., A. Li, M. Song, L. Ma, and J. Jiang (2010), Reconstruction of NDVI timeseries datasets of MODIS based on Savitzky-Golay filter, J. Remote Sens. 14, 4, 725-741.

Bonettini, S., and V. Ruggiero (2011), An alternating extragradient method for total variation-based image restoration from Poisson data, Inverse Probl. 27, 9, 095001, DOI: 10.1088/0266-5611/27/9/095001.

Boudraa, A.O., J.C. Cexus, and Z. Saidi (2004), EMD-based signal noise reduction, Int. J. Signal Process. 1, 1, 33-37.

Chambolle, A. (2004), An algorithm for total variation minimization and applications, J. Math. Imaging Vis. 20, 1-2, 89-97, DOI: 10.1023/B:JMIV. $0000011325.36760 .1 \mathrm{e}$.

Chambolle, A., and P.-L. Lions (1997), Image recovery via total variation minimization and related problems, Numer. Math. 76, 2, 167-188, DOI: 10.1007/ s002110050258.

Chambolle, A., V. Caselles, D. Cremers, M. Novaga, and T. Pock (2010), An introduction to total variation for image analysis. In: M. Fornasier (ed.), Theoretical Foundations and Numerical Methods for Sparse Recovery, Radon Series on Computational and Applied Mathematics, Vol. 9, de Gruyter, Berlin, 263-340,

Chan, T., S. Esedoglu, F. Park, and A. Yip (2006), Total variation image restoration: Overview and recent developments. In: N. Paragios, Y. Chen, and O. Faugeras (eds.), Handbook of Mathematical Models in Computer Vision, Springer, New York, 17-32.

Chen, D., and L. Cheng (2012), Spatially adapted total variation model to remove multiplicative noise, IEEE Trans. Image Process. 21, 4, 1650-1662, DOI: 10.1109/TIP.2011.2172801.

Chen, J., P. Jönsson, M. Tamura, Z. Gu, B. Matsushita, and L. Eklundh (2004), A simple method for reconstructing a high-quality NDVI time-series data set based on the Savitzky-Golay filter, Remote Sens. Environ. 91, 3-4, 332344, DOI: $10.1016 /$ j.rse.2004.03.014.

Chen, Z., and J. Shu (2011), Remote sensing image merging based on SavitzkyGolay method, Geogr. Geo-Inf. Sci. 27, 2, 29-33. 
Člupek, M., P. Matějka, and K. Volka (2007), Noise reduction in Raman spectra: Finite impulse response filtration versus Savitzky-Golay smoothing, $J$. Raman Spectrosc. 38, 9, 1174-1179, DOI: 10.1002/jrs.1747.

Dong, F.F., and Z. Liu (2009), A new gradient fidelity term for avoiding staircasing effect, J. Comput. Sci. Technol. 24, 6, 1162-1170, DOI: 10.1007/s11390009-9289-1.

Ferahtia, J., K. Baddari, N. Djarfour, and A.K. Kassouri (2010), Incorporation of a non-linear image filtering technique for noise reduction in seismic data, Pure Appl. Geophys. 167, 11, 1389-1404, DOI: 10.1007/s00024-010-0101-5.

Gong, C. (2012), Method research of the seismic data denoising based on total variation principle, MSc. Thesis, Chengdu University of Technology, Chengdu, China, 46 pp.

Guiñón, J.L., E. Ortega, J. García-Antón, and V. Pérez-Herranz (2007), Moving average and Savitzki-Golay smoothing filters using Mathcad. In: Proc. Int. Conf. on Engineering Education (ICEE 2007), 3-7 September 2007, Coimbra, Portugal.

Hargittai, S. (2005), Savitzky-Golay least-squares polynomial filters in ECG signal processing, Comput. Cardiol. 32, 763-766, DOI: 10.1109/CIC.2005. 1588216.

Wayt, H.J., and T.R. Khan (2007), Integrated Savitzky-Golay filter from inverse Taylor series approach. In: Proc. 15th Int. Conf. on Digital Signal Processing, 1-4 July 2007, Cardiff, Great Britain, 375-378, DOI: 10.1109/ICDSP. 2007.4288597.

Huang, Y.H., J.H. Wang, D. Jiang, and Q. Zhou (2009), Reconstruction of MODISEVI time-series data with S-G filter, Geomat. Inf. Sci. Wuhan Univ. 34, 12, 1440-1444.

Li, M., and J. Liu (2011), Reconstructing vegetation temperature condition index based on the Savitzky-Golay filter. In: D. Li, Y. Liu, and Y. Chen (eds.), Computer and Computing Technologies in Agriculture IV, IFIP Advances in Information and Communication Technology, Vol. 346, 629-637, DOI: 10.1007/978-3-642-18354-6_74.

Li, X., and N.M. Bilgutay (1993), Wiener filter realization for target detection using group delay statistics, IEEE Trans. Signal Process. 41, 6, 2067-2074, DOI: 10.1109/78.218136.

Nie, P. (2012), The study and application of noise suppression method in seismic exploration - Regularization constrained Wiener filter and directional derivative trace transform, Ph.D. Thesis, Jilin University, Jilin, China.

O'Haver, T. (2012), An introduction to signal processing with applications in chemical analysis, http://terpconnect.umd.edu/ toh/spectrum/TOC.html, last updated 8 May.

O’Haver, T. (2013), A pragmatic introduction to signal processing with applications in chemical analysis, http://terpconnect.umd.edu/ toh/spectrum/TOC.html, last updated 28 July. 
Qu, Y., J.X. Cao, H.D. Zhu, and C. Ren (2011), An improved total variation technique for seismic image denoising, Acta Petrolei Sin. 32, 815-819.

Rudin, L.I., S. Osher, and E. Fatemi (1992) Nonlinear total variation based noise removal algorithms, Physica D 60, 1-4, 259-268, DOI: 10.1016/01672789(92)90242-F.

Ruffin, C., and R.L. King (1999), The analysis of hyperspectral data using SavitzkyGolay filtering - Theoretical basis (Part 1). In: Conf. IEEE Int. Geoscience and Remote Sensing Symposium, Hamburg, Germany, 756-758, DOI: 10.1109/IGARSS.1999.774430.

Savitzky, A., and M.J.E. Golay (1964), Smoothing and differentiation of data by simplified least squares procedures, Anal. Chem. 36, 8, 1627-1639, DOI: 10.1021/ac60214a047.

Schafer, R.W. (2011), What is a Savitzky-Golay filter? IEEE Signal Process. Mag. 28, 4, 111-117, DOI: 10.1109/MSP.2011.941097.

Selesnick, I.W., and İ. Bayram (2010), Total variation filtering, OpenStax-CNX module: m31292, http://cnx.org/content/m31292/1.1.

Vaiphasa, C. (2006), Consideration of smoothing techniques for hyperspectral remote sensing, ISPRS J. Photogramm. Remote Sens. 60, 2, 91-99, DOI: 10.1016/j.isprsjprs.2005.11.002.

Vivó-Truyols, G., and P.J. Schoenmakers (2006), Automatic selection of optimal Savitzky-Golay smoothing, Anal. Chem. 78, 13, 4598-4608, DOI: 10.1021/ ac0600196.

Vogel, C.R., and M.E. Oman (1996), Iterative methods for total variation denoising, SIAM J. Sci. Comput. 17, 1, 227-238, DOI: 10.1137/0917016.

Xu, J., H. Jiang, L. Xu, L. Jiang, T. Qian, and C. Feng (2011), Pulse wave processing and analysis based on smoothing filter, Chin. J. Sci. Instr. 32, 334-337.

Zehtabian, A., and H. Hassanpour (2011), Optimized singular vector denoising approach for speech enhancement, Iran. J. Ener. Environ. 2, 2, 166-180.

Zhou, W., and Q. Li (2013), Adaptive total variation regularization based scheme for Poisson noise removal, Math. Method. Appl. Sci. 36, 3, 290-299, DOI: 10.1002/mma.2587.

Received 3 September 2013 Received in revised form 11 January 2015 Accepted 9 February 2015 